\title{
The GAIA (CLL13) trial - an international, randomized, four-arm study for first line treatment of physically fit CLL patients without del17p or TP53 mutation
}

\author{
Von Tresckow $\mathrm{J}^{1 *}$, Bahlo $\mathrm{J}^{1}$, Niemann $\mathrm{CU}^{2}$, Kater $\mathrm{AP}^{3}$, Fink $\mathrm{AM}^{1}$, Fischer $\mathrm{K}^{1}$, Ritgen $\mathrm{M}^{4}$, Kreuzer $\mathrm{KA}^{1}$, Stilgenbauer $\mathrm{S}^{5}$, van Oers $\mathrm{R}^{3}$, \\ Hartmann Geisler $\mathrm{C}^{2}$, Hallek $\mathrm{M}^{6}$ and Eichhorst $\mathrm{B}^{1}$ \\ ${ }^{1}$ Department I of Internal Medicine and Center of Integrated Oncology Cologne-Bonn, German CLL Study Group, University of Cologne, Germany \\ ${ }^{2}$ Department of Hematology, Rigshospitalet, Copenhagen University Hospital, Nordic CLL Study Group, Denmark \\ ${ }^{3}$ Department of Hematology, Academic Medical Center, Amsterdam, the Netherlands, on behalf of the HOVON CLL Working Group \\ ${ }^{4}$ Department of Internal Medicine II, Campus Kiel, University of Schleswig-Holstein, Germany \\ ${ }^{5}$ Department III of Internal Medicine, University Hospital Ulm, Germany \\ ${ }^{6}$ Department I of Internal Medicine and Center of Integrated Oncology Cologne-Bonn, CECAD Cluster of Excellence at the University of Cologne, Clinical \\ Research Unit (KFO) 286, German CLL Study Group, University of Cologne, Cologne, Germany
}

We report about a phase III, international, randomized, prospective, open-label study (GAIA or CLL13 trial), designed and executed as investigator initiated trial of the German CLL Study Group (GCLLSG) in cooperation with the Nordic CLL Study Group, HOVON, SAKK, Cancer Trials Ireland and the Israeli CLL Study Group.

Physically fit patients with previously untreated chronic lymphocytic leukemia (CLL) without del(17p) or TP53 mutation are randomized into four different treatment arms with 230 patients in each of them (see figure 1). 160 trial sites within 11 countries in Europe and Israel will be active in recruiting 920 patients in total. Recruitment started in December 2016 and is estimated to take about 33 month. The final analysis of the co-primary endpoint MRD negativity and the interim analysis for the PFS endpoint are expected to take place 49 months after the first patient entered the trial, which was on December $14^{\text {th }} 2016$. The final PPS endpoint analysis is expected to be reached 73 months after trial initiation, which will also be the formal end of trial.

The study was designed to test whether different combinations of novel, chemotherapy-free regimes are superior to chemoimmunotherapy, the current standard of care.

Fludarabine, cyclophosphamide and rituximab (FCR) is the most effective regimen known so far in the tested patient group [1,2]. Efficacy varies substantially depending on prognostic risk-groups. Due to the high risk of severe neutropenias and infections with FCR, bendamustine and rituximab (BR) as alternative regimen was chosen as comparator arm for patients aged $>65$ years according to current treatment guidelines.

Nevertheless, conventional chemoimmunotherapies are associated with side effects caused by the general cytotoxicity of the chemotherapy. Novel regimens that have both, an increased efficacy as well as a superior toxicity profile, are therefore warranted.

Venetoclax (GDC-0199/ABT-199), a BCL2 antagonist, showed striking activity with tumor lysis syndrome as serious toxicity in CLL therapy. Venetoclax is approved for the treatment of relapsed CLL by the FDA and EMA since 2016. In a phase II study, several patients treated with the combination of venetoclax and rituximab in relapsed/ refractory CLL even achieved MRD negativity [3].

This indicates that combinations of venetoclax and CD20antibodies have the potential to induce higher rates of minimal residual disease (MRD) negativity in frontline therapy of CLL with concomitantly less toxicity. Thus, chemotherapy might be replaced by venetoclax.

The novel, glycoengineered, anti-CD20-antibody obinutuzumab has become the standard of care therapy in first line treatment of elderly patients with CLL and coexisting conditions as its combination with chlorambucil proved to be safe and lead to markedly improved response rates as well as PFS times in comparison to chlorambucil alone or combined with rituximab in the CLL11 trial [4].

Furthermore, venetoclax and obinutuzumab demonstrated synergistic activity in a preclinical study of a murine Non-Hodgkin lymphoma xenograft model, and additive activity in a CLL lymph node model [5]. The combination appears highly efficacious and well tolerable in the firstline treatment of CLL patients with coexisting conditions whilst the toxicity profile of both drugs compares favorably to those of the chemotherapies currently used in the treatment of CLL [6].

We therefore hypothesize for one of the two co-primary endpoints that a combination of obinutuzumab plus venetoclax (GVe) increases the proportion of patients with no detectable MRD in peripheral blood at month 15 in comparison to standard chemoimmunotherapy.

Ibrutinib, a selective, irreversible inhibitor of Bruton's Tyrosine Kinase (BTK), showed excellent responses and a safe toxicity profile, even in combination with BR [7]. In comparison to chlorambucil,

Correspondence to: Dr. Julia v. Tresckow, Specialist, Study Physician, Department I of Internal Medicine, German CLL Study Group (DCLLSG), University Hospital of Cologne, Kerpener Str. 62, D-50937 Cologne, Germany, Tel: +49 221 478-88220, Fax: +49 221478 1423777, E-mail: julia.von-tresckow@uk-koeln.de

Received: September 08, 2017; Accepted: September 26, 2017; Published: September 28, 2017 


\section{CLL13/GAIA TRIAL}

Standard chemoimmunotherapy vs. venetoclax + rituximab vs. venetoclax + obinutuzumab (GA101) vs. venetoclax + ibrutinib + obinutuzumab (GA101)

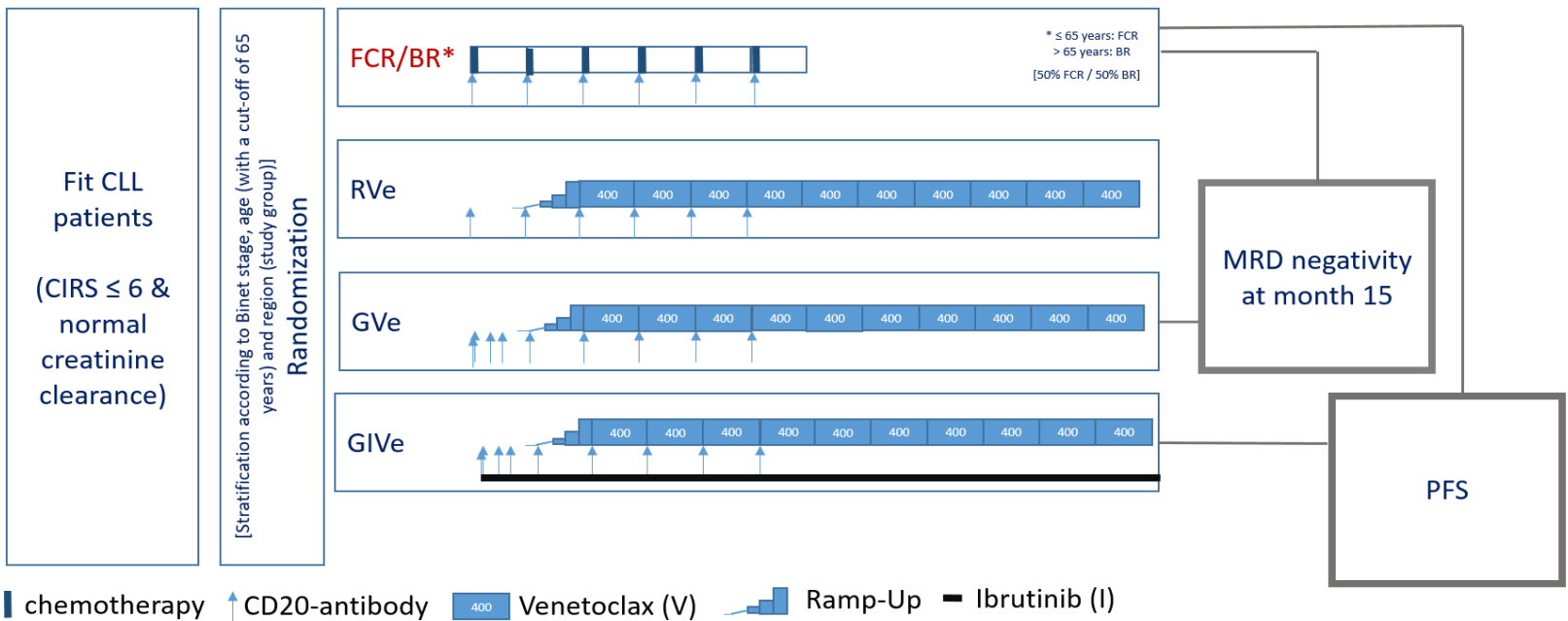

Figure 1. Overview of the four treatment arms of the study: Standard chemoimmunotherapy (FCR/BR) vs. venetoclax + rituximab (RVe) vs. venetoclax + obinutuzumab (GVe) vs. venetoclax + ibrutinib + obinutuzumab (GIVe). The two co-primary endpoints, MRD and PFS, are indicated for the comparison of the standard chemoimmunotherapy arm vs the GVe arm and the GIVe arm, respectively.

ibrutinib was superior in frontline therapy of the elderly with regard to progression free survival (PFS) and overall survival. Ibrutinib is approved for frontline and relapse treatment of CLL by the FDA and EMA since 2016.

The combination of ibrutinib and venetoclax showed synergy in primary CLL cells [8]. Consequently, a combination of these targeted drugs with obinutuzumab is tested in the fourth arm of the trial with the aim to demonstrate superiority in terms of improved PFS in comparison with standard chemoimmunotherapy. This is the second co-primary endpoint of the trial.

Secondary endpoints include comparisons of MRD and PFS for the other treatment arms as well as the overall survival, safety parameters as well as health-related quality of life.

In conclusion, the results of this phase III study are likely to change first line treatment for physically fit CLL patients without del17p or TP53 mutation to more efficacious, less toxic, chemotherapy-free treatment regimes.

\section{References}

1. Fischer K, Bahlo J, Fink AM, Goede V, Herling CD, et al. (2016) Long-term remissions after FCR chemoimmunotherapy in previously untreated patients with CLL: updated results of the CLL8 trial. Blood 127: 208-215. [Crossref]
2. Thompson PA, Tam CS, O'Brien SM, Wierda WG, Stingo F, et al. (2016) Fludarabine, cyclophosphamide, and rituximab treatment achieves long-term disease-free survival in IGHV-mutated chronic lymphocytic leukemia. Blood 127: 303-309. [Crossref]

3. Seymour JF, Ma S, Brander DM, Choi MY, Barrientos J, et al. (2017) Venetoclax plus rituximab in relapsed or refractory chronic lymphocytic leukaemia: a phase $1 \mathrm{~b}$ study. Lancet Oncol 18: 230-240. [Crossref]

4. Goede V, Fischer K, Busch R, Engelke A, Eichhorst B, et al. (2014) Obinutuzumab plus chlorambucil in patients with CLL and coexisting conditions. N Engl J Med 370: 1101-1110. [Crossref]

5. Thijssen R, Slinger E, Weller K, Geest CR, Beaumont T, et al. (2015) Resistance to ABT-199 induced by microenvironmental signals in chronic lymphocytic leukemia can be counteracted by CD20 antibodies or kinase inhibitors. Haematologica 100: e302306. [Crossref]

6. Fischer K, Al-Sawaf O, Fink AM, Dixon M, Bahlo J, et al. (2017) Venetoclax and obinutuzumab in chronic lymphocytic leukemia. Blood 29: 2702-2705. [Crossref]

7. Chanan-Khan A, Cramer P, Demirkan F, Fraser G, Silva RS, et al. (2016) Ibrutinib combined with bendamustine and rituximab compared with placebo, bendamustine, and rituximab for previously treated chronic lymphocytic leukaemia or small lymphocytic lymphoma (HELIOS): a randomised, double-blind, phase 3 study. Lancet Oncol 17: 200-211. [Crossref]

8. Deng J, Isik E, Fernandes SM, Brown JR, Letai A, et al. (2017) Bruton's tyrosine kinase inhibition increases BCL-2 dependence and enhances sensitivity to venetoclax in chronic lymphocytic leukemia. Leukemia 31: 2075-2084. [Crossref]

Copyright: $\odot 2017$ von Tresckow J. This is an open-access article distributed under the terms of the Creative Commons Attribution License, which permits unrestricted use, distribution, and reproduction in any medium, provided the original author and source are credited. 